\title{
Multifacility Location Problem using Scaled Conjugate Gradient Algorithm under Triangular Area Constraints
}

\author{
G.M. Nasira \\ Professor \& Vice Principal \\ Sasurie College of Engineering \\ Viyayamangalam, Tirupur (Dt), \\ Tamil Nadu, India
}

\author{
T.S.S. Balaji \\ Executive Director \\ Sasurie Institutions \\ Vijayamangalam, Tirupur (Dt), \\ Tamil Nadu, India
}

\begin{abstract}
The problem of finding the multiple locations for new facilities with respect to the multiple existing facilities in a given environment is known as Multifacility Location Problem (MLP).Every location problem is normally bounded by some sort of area constraint. But the fact that much of the work carried out in the literature has almost neglected the area constraint which has motivated us to work on Multifacility Location Problem taking the area constraint into consideration. The mathematical model of the multifacility location problem with area constraint has been developed and the solution has been obtained using Kuhn-Tucker theory. This mathematical analysis and solution procedure is highly complex and time consuming. Hence, an attempt has been made to get the solution of a complex, constrained multifacility location problem using Scaled Conjugate Gradient Algorithm (SCGA) in Artificial Neural Networks (ANN). With the help of Numerical examples, it has been established that the solution obtained through ANN model compares well within the acceptable limits with those obtained through analytical method.
\end{abstract}

\section{Indexterms}

Multifacility Location Problem, Area Constraint, Kuhn-Tucker theory, Artificial Neural Networks (ANN), Scaled Conjugate Gradient Algorithm (SCGA).

\section{INTRODUCTION}

A number of real life situations involve the problem of locating new facilities with respect to the existing facilities. The multifacility location problems refer to the process of finding the multiple locations for the new facilities with respect to the existing multiple facilities in the prevailing environment. The past investigations into Multifacility Location problems have not taken the availability of area for locating the new facilities into consideration. In this regard we may mention the work of Cabot, Francis and Stray[1],Pritsker and Ghare[2], Kuhn and Kuenne [3], Eyster and White[4] and McHose[5]. Hence in our study of MLP, we have taken the area constraints into consideration. We have considered a deterministic model with the concept of Euclidean distance between facilities with the requirement that the new facilities must be located in a restricted area of triangular shape. This leads to linear constraints. We have solved the problem by using Kuhn-Tucker condition and illustrated the solution procedure of the problem by using some numerical data.

\subsection{Applications of ANN}

The training and testing are two important phases in the development of ANN. The large number of training data sets containing input data and target output are to be fed to the ANN in the training phase. The neural networks create connections and learn patterns based on these data sets as pointed out by Rumelhart [7].Each pattern creates a unique configuration of network structure with a unique set of connection strengths or weights. Similarly, if the neural network does not match the pattern within the given tolerance, it will adjust and try again. A neural network adapts in changing inputs and learns trends from data. Each connection weight builds on previous decision nodes, propagating down to a final decision. After the neural network reaches a final decision, it compares its answer against an answer provided in the training set as target output. If there is a match, within a predefined tolerance, the neural network stores these connection weights as successful. If the decision outcome is outside the tolerance, then the neural network cycles through the training set again. A neural network may cycle thousands of times to reach an acceptable tolerance. The optimal network performance is quite dependent upon making the proper network structure [8]. We have first solved a set of multifacility location problems using the analytical method and then we have again solved the same set of problems through ANN technique using SCGA training algorithm. It is found that the solutions obtained through prediction by using this training algorithm compare well with those obtained using analytical method. The prediction error percentage vary between $0-13 \%$ which is well within the acceptable limits. Further, the graphical representation of the results obtained analytically (Anal) and through ANN techniques have also been made to have visual clarity in comparison.

\subsection{PROBLEM FORMULATION ANDANALYTICAL SOLUTION}

$$
\begin{aligned}
& \text { The MLP with triangular area constraint can be stated as: } \\
& \text { Minimize } \mathrm{f}\left(\left(\mathrm{x}_{1}, \mathrm{y}_{1}\right),\left(\mathrm{x}_{2}, \mathrm{y}_{2}\right), \ldots \ldots,\left(\mathrm{x}_{\mathrm{n}}, \mathrm{y}_{\mathrm{n}}\right)\right) \\
& =\sum_{1 \leq \mathrm{j}<\mathrm{k} \leq \mathrm{n}(\mathrm{j} \neq \mathrm{k})} \mathrm{v}_{\mathrm{jk}}\left[\left(\mathrm{x}_{\mathrm{j}}-\mathrm{x}_{\mathrm{k}}\right)^{2}+\left(\mathrm{y}_{\mathrm{j}}-\mathrm{y}_{\mathrm{k}}\right)^{2}+\varepsilon\right] \\
& +\sum_{\mathrm{i}=1 \mathrm{j}=1}^{\mathrm{m}} \sum_{\mathrm{j}}^{\mathrm{n}} \mathrm{w}_{\mathrm{ji}}\left[\left(\mathrm{x}_{\mathrm{j}}-\hat{\mathrm{x}}_{\mathrm{i}}\right)^{2}+\left(\mathrm{y}_{\mathrm{j}}-\hat{\mathrm{y}}_{\mathrm{i}}\right)^{2}+\varepsilon\right]
\end{aligned}
$$

Subject to: 


$$
\begin{aligned}
& a x_{j}+b_{j}+c \leq 0 \quad(a, b>0 \text { and } c<0) \\
& \text { and } x_{j} \geq 0, y_{j} \geq 0,(j=1,2, \ldots, n) \\
& \text { where } \\
& \mathrm{n}=\text { number of new facilities to be located, } \\
& \mathrm{m}=\text { number of existing facilities, } \\
& \left(x_{j}, y_{j}\right)=\text { co-ordinates of the } j^{\text {th }} \text { new facility, } \\
& \hat{\left(x_{i}, y_{i}\right)}=\text { co-ordinates of the } i^{\text {th }} \text { existing facility, } \\
& \mathrm{v}_{\mathrm{jk}}=\text { cost per unit distance between new } \\
& \text { facility } \mathrm{j} \text { and new facility } \mathrm{k} \text {, } \\
& \mathrm{v}_{\mathrm{jj}} \quad=0 \text {, and } \\
& \mathrm{w}_{\mathrm{ji}} \quad=\text { cost per unit distance between new } \\
& \text { facility } \mathrm{j} \text { and existing facility } \mathrm{i} \text {. }
\end{aligned}
$$

We shall use Kuhn-Tucker conditions to get the solution of the problem for which we construct the auxiliary function as follows:

$$
\begin{aligned}
& h(x, y)= f(x, y)-\lambda\left[(a x+b y+c)+\eta^{2}\right. \\
& \text { i.e., } h\left(\left(x_{1}, y_{1}\right),\left(x_{2}, y_{2}\right), \ldots .,\left(x_{n}, y_{n}\right)\right) \\
&= f\left(\left(x_{1}, y_{1}\right),\left(x_{2}, y_{2}\right), \ldots .,\left(x_{n}, y_{n}\right)\right) \\
&-\sum_{j=1}^{n} \lambda_{j}\left[\left(a_{j}+b_{j}+c\right)+\eta_{j}^{2}\right]
\end{aligned}
$$

where $\eta_{j}^{2}$ are the artificial variables and are given by

$$
\eta_{j}^{2}=-\left(a x_{j}+b_{j}+c\right) \text {. }
$$

Now by using Kuhn-Tucker theory we get the following set of necessary conditions:

$$
\begin{aligned}
& \frac{\delta h}{\delta x_{j}}=\frac{\delta f}{\delta x_{j}}-a \lambda_{j}=0 \\
& \frac{\delta h}{\delta y_{j}}=\frac{\delta f}{\delta y_{j}}-b \lambda_{j}=0 \\
& \lambda_{j}\left(a x_{j}+b y_{j}+c\right)=0 \\
& a x_{j}+b y_{j}+c \leq 0
\end{aligned}
$$

and $\lambda_{j} \geq 0 \quad(j=1,2, \ldots \ldots, n)$. We may note here that the necessary conditions for the occurrence of the minimum are also sufficient in view of the convexity of the objective function. In view of

$$
\begin{aligned}
& \frac{\delta f}{\delta x_{j}}=\sum_{k=1 \neq j}^{n} \frac{v_{j k}^{\prime}\left(x_{j}-x_{k}\right)}{D_{j k}}+\sum_{i=1}^{m} \frac{w_{j i}\left(x_{j}-x_{i}\right)}{E_{j i}} \\
& \frac{\delta f}{\delta y_{j}}=\sum_{k=1 \neq j}^{n} \frac{v_{j k}^{\prime}\left(y_{j}-y_{k}\right)}{D_{j k}}+\sum_{i=1}^{m} \frac{w_{j i}\left(y_{j}-y_{i}\right)}{E_{j i}}
\end{aligned}
$$

$$
\begin{aligned}
& D_{j k}=\left[\left(x_{j}-x_{k}\right)^{2}+\left(y_{j}-y_{k}\right)^{2}+\varepsilon\right]^{1 / 2} \\
& E_{j i}=\left[\left(x_{j}-x_{i}\right)^{2}+\left(y_{j}-y_{i}\right)^{2}+\varepsilon\right]^{1 / 2} \\
& \text { and } v^{\prime} j_{k}=\left\{\begin{array}{l}
v_{j k}, k>j \\
v_{k j}, k<j .
\end{array}\right.
\end{aligned}
$$

There are only two possible cases to be examined, viz., (i) when $\lambda j=0$ and (ii) when $\lambda_{j} \neq 0$. First we consider the case when $\lambda j=0$.

Case $-\mathrm{I}: \lambda_{\mathrm{j}}=0(\mathrm{j}=1,2, \ldots \ldots, \mathrm{n})$.

As $\lambda_{\mathrm{j}}=0$, the equations (2.5) and (2.6) reduce to $\frac{\delta f}{\delta x_{j}}=0$ and $\frac{\delta f}{\delta y_{j}}=0$ respectively which in view of (2.9) and (2.10) lead respectively to

$$
\sum_{k=1 \neq j}^{n} \frac{v_{j k}^{\prime}\left(x_{j}-x_{k}\right)}{D_{j k}}+\sum_{i=1}^{m} \frac{w_{j i}\left(x_{j}-x_{i}\right)}{E_{j i}}=0
$$

$\sum_{k=1 \neq j}^{n} \frac{v_{j k}\left(y_{j}-y_{k}\right)}{D_{j k}}+\sum_{i=1}^{m} \frac{w_{j i}\left(y_{j}-y_{i}\right)}{E_{j i}}=0$

The simplification of (2.14) and(2.15) will result in

$$
x_{j}=\frac{\sum_{k=1 \neq j}^{n} \frac{v^{\prime}{ }_{j k} x_{k}}{D_{j k}}+\sum_{i=1}^{m} \frac{w_{j i} x_{i}}{E_{j i}}}{\sum_{k=1}^{n} \frac{v^{\prime} j k}{D_{j k}}+\sum_{i=1}^{m} \frac{w_{j i}}{E_{j i}}}
$$

and

$$
y_{j}=\frac{\sum_{k=1 \neq j}^{n} \frac{v^{\prime} j k y_{k}}{D_{j k}}+\sum_{i=1}^{m} \frac{w_{j i} y_{i}}{E_{j i}}}{\sum_{k=1}^{n} \frac{v^{\prime} j k}{D_{j k}}+\sum_{i=1}^{m} \frac{w_{j i}}{E_{j i}}}
$$

To solve the set of non-linear equations represented by (2.16) and (2.17) we use the following iterative scheme.

Iterative Scheme: The equations (2.16) and (2.17) can be written as :

$$
\mathrm{x}_{\mathrm{j}}=\mathrm{F}_{\mathrm{j}}\left(\mathrm{x}_{1}, \mathrm{x}_{2}, \ldots, \mathrm{x}_{\mathrm{n}}, \mathrm{y}_{1}, \mathrm{y}_{2}, \ldots ., \mathrm{y}_{\mathrm{n}}\right)
$$


and $y_{j}=G_{j}\left(x_{1}, x_{2}, \ldots ., x_{n}, y_{1}, y_{2}, \ldots ., y_{n}\right)$

Starting with the initial solution where the superscripts denote the iteration number, we take the initial solution as

$$
\mathrm{x}_{\mathrm{j}}^{(0)}=\frac{\sum_{\mathrm{i}=1}^{\mathrm{m}} \mathrm{w}_{\mathrm{ji}} \mathrm{x}_{\mathrm{i}}^{\wedge}}{\sum_{\mathrm{k}=1 \neq \mathrm{j}}^{\mathrm{n}} \mathrm{v}^{\prime} \mathrm{jk}_{\mathrm{i}=1}+\sum_{j i}^{\mathrm{m}} \mathrm{w}_{\mathrm{ji}}}
$$

and

$$
y_{j}^{(0)}=\frac{\sum_{i=1}^{m} w_{j i} y_{i}}{\sum_{k=1 \neq j}^{n} v^{\prime}{ }_{j k}+\sum_{i=1}^{m} w_{j i}}
$$

Now the solution $\left(x_{j}, y_{j}\right)(j=1,2, \ldots, n)$ obtained by the above iterative scheme has to be tested whether it satisfies the constraint (2.2). If it satisfies (2.2), the problem is solved and $\left(\mathrm{x}_{\mathrm{j}}, \mathrm{y}_{\mathrm{j}}\right)$ $(j=1,2, \ldots \ldots, n)$ give the optimum location for the new facilities. If this solution does not satisfy (2.2), we have the only alternative of considering the case when $\lambda_{\mathrm{j}} \neq 0 \quad(\mathrm{j}=1,2, \ldots \ldots, \mathrm{n})$ and in this case all the new facilities lie on the boundary.

Case -II: $\lambda_{\mathrm{j}} \neq 0(\mathrm{j}=1,2, \ldots \ldots \ldots, \mathrm{n})$.

Since $\lambda_{j} \neq 0$, the equation (2.5a) takes the form $\mathrm{ax}_{\mathrm{j}}+\mathrm{by}_{\mathrm{j}}+\mathrm{c}=0$ which gives

$$
y_{j}=-\frac{c}{b}-\frac{a}{b} x \text { j }
$$

The Equations (2.4) and (2.5) lead to

$$
\frac{\delta f}{\delta x_{j}}=\frac{a}{b} \frac{\delta f}{\delta y_{j}}
$$

In view of (2.6) and (2.7) the equation (2.18) takes the form

$\sum_{k=1 \neq j}^{n} \frac{v_{j k}^{\prime}\left(x_{j}-x_{k}\right)}{D_{j k}}+\sum_{i=1} \frac{w_{j i}\left(x_{j}-x_{i}\right)}{E_{j i}}$

$=\frac{a}{b}\left[\sum_{k=1 \neq j}^{n} \frac{v_{j k}^{\prime}\left(y_{j}-y_{k}\right)}{D_{j k}}+\sum_{i=1} \frac{w_{j i}\left(y_{j}-y_{i}\right)}{E_{j i}}\right]$

Further simplification of (2.26) by using (2.11) and (2.12) and value of yj or yk from (2.22) finally leads to

$$
\begin{gathered}
\mathrm{x}_{\mathrm{j}}=\frac{\mathrm{P}}{\mathrm{Q}} \\
\text { where }
\end{gathered}
$$

$$
P=\frac{b}{\sqrt{a^{2}+b^{2}}} \sum_{k=1}^{n} \frac{j}{\sqrt{\left(x_{j}-x_{k}\right)^{2}+\varepsilon \frac{b^{2}}{a^{2}+b^{2}}}}
$$$$
+\frac{b^{3}}{a^{2}+b^{2}} \sum_{i=1}^{m} \frac{w_{j i} x_{i}}{T_{j i}}-\frac{a b^{2}}{a^{2}+b^{2}} \sum_{i=1}^{m_{j i}} \frac{w_{j i} y_{i}}{T_{j i}}
$$$$
-\frac{a b c}{a^{2}+b^{2}} \sum_{i=1}^{m} \frac{w_{j i}}{T_{j i}},
$$

$$
Q=\frac{b}{\sqrt{a^{2}+b^{2}}} \sum_{k=1 \neq j}^{n} \frac{v^{\prime} j k}{\sqrt{\left(x_{j}-x_{k}\right)^{2}+\varepsilon \frac{b^{2}}{a^{2}+b^{2}}}}
$$

$$
+b \sum_{i=1}^{m} \frac{{ }^{\mathrm{w}} \mathrm{ji}}{\mathrm{T}_{\mathrm{ji}}}
$$

and $\mathrm{T}_{\mathrm{ji}}=\left[\mathrm{b}^{2}\left(\mathrm{x}_{\mathrm{j}}-\hat{\mathrm{x}}_{\mathrm{i}}\right)^{2}+\left(a \mathrm{x}_{\mathrm{j}}+\mathrm{b} \mathrm{y}_{\mathrm{i}}+\mathrm{c}\right)^{2}+\varepsilon \mathrm{b}^{2}\right]^{1 / 2}$.

By similar arguments we obtain the relations for yj as

$$
\mathrm{y}_{\mathrm{j}}=\frac{\mathrm{R}}{\mathrm{S}}
$$

$$
R=\frac{a}{\sqrt{a^{2}+b^{2}}} \sum_{k=1 \neq j}^{n} \frac{v_{j k}^{\prime} y_{k}}{\sqrt{\left(y_{j}-y_{k}\right)^{2}+\varepsilon \frac{a^{2}}{a^{2}+b^{2}}}}
$$

$$
\begin{aligned}
& +\frac{a^{3}}{a^{2}+b^{2}} \sum_{i=1}^{m} \frac{w_{j i} y_{i}}{K_{j i}}-\frac{a^{2} b}{a^{2}+b^{2}} \sum_{i=1}^{m} \frac{w_{j i} x_{i}}{K_{j i}} \\
& -\frac{a b c}{a^{2}+b^{2}} \sum_{i=1}^{m} \frac{w_{j i}}{K_{j i}},
\end{aligned}
$$

$$
S=\frac{a}{\sqrt{a^{2}+b^{2}}} \sum_{k=1 \neq j}^{n} \frac{v_{j k}^{\prime}}{\sqrt{\left(y_{j}-y_{k}\right)^{2}+\varepsilon \frac{a^{2}}{a^{2}+b^{2}}}}+a \sum_{i=1}^{m} \frac{\mathrm{K}_{j i}}{K_{j i}}
$$

and

$$
K_{j i}=\left[a^{2}\left(y_{j}-y_{i}\right)^{2}+\left(b y_{j}+a x_{i}+c\right)^{2}+\varepsilon a^{2}\right]^{1 / 2} \text {. }
$$

To solve the set of non-linear equations represented by (2.25) or (2.26) we use the following iterative scheme :

Iterative Scheme:

Starting with the initial solution 
$\left(\mathrm{x}_{1}{ }^{(0)}, \mathrm{x}_{2}{ }^{(0)}, \ldots ., \mathrm{x}_{\mathrm{n}}{ }^{(0)}\right)$

we form the successive approximate solutions from the relations given by :

$\mathrm{x}_{\mathrm{j}}^{(\mathrm{N}+1)}=\mathrm{H}_{\mathrm{j}}\left(\mathrm{x}_{1}{ }^{(\mathrm{N})}, \mathrm{x}_{2}{ }^{(\mathrm{N})}, \ldots, \mathrm{x}_{\mathrm{n}}{ }^{(\mathrm{N})}\right)$

where the superscripts denote the iteration number. It may be noted here that after finding the values of all $\mathrm{x}_{\mathrm{j}}(\mathrm{j}=1,2, \ldots, \mathrm{n})$ by using the iterative scheme (2.27), one need not calculate the values of all corresponding $y_{j}(j=1,2, \ldots, n)$ by using the iterative scheme as these can be found directly from the equation (2.22) with the help of the determined values of all $x_{j}(j=1,2, \ldots, n)$.

\section{MULTIFACILITY LOCATION PROBLEM WITH TRIANGULAR AREA CONSTRAINT}

The analytical solution method for MLP described in Section II is tedious and time consuming. Hence, an attempt has been made to solve the MLP with triangular area constraint using ANN techniques with SCGA[7].

\subsection{Solutions of MLP using Scaled Conjugate Gradient Algorithm (SCGA)}

The network that we have used for the training has the structure as shown in Fig. 3.1.1 consisting of (i) One Input Layer having 10 neurons, (ii) Two Hidden Layers having 50 neurons in the first Hidden Layer and 25 neurons in the second Hidden Layer and (iii) One Output Layer having 4 neurons. It has been seen that the training of the net using the algorithm has been completed well in time after an optimal number of epochs and the performance of training the net is found to be $99.99 \%$ (given in Fig. 3.1.2).

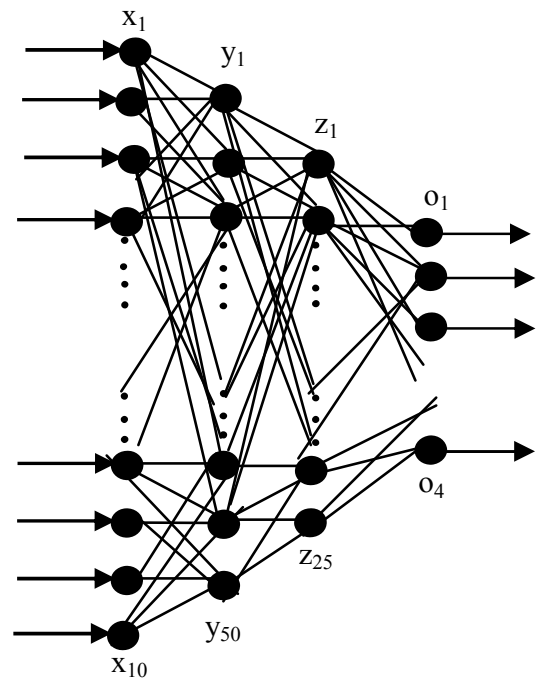

Input Hidden Hidden Output

Layer Layer 1 Layer 2 Layer

Fig. 3.1.1 Network Structure $(10,50,25,4)$

We consider the example involving 2 new and 2 existing facilities where the new facilities are to be located in a triangular area given by $\mathrm{xi}+\mathrm{yi}-\mathrm{c}<=0$, where $\mathrm{c}$ will have different values for different problems. By using these input parameters we have found the locations of 2 new facilities viz. (i) (x1, y1) and (ii) (x2, y2) as the solution. A set of 75 numerical examples has been considered here to find the solution using SCGA training technique. Each of these 75 data sets has 10 input values and 4 output values which have been used as input and target output respectively to the network for training. We have used $1 \times 10-5$ as the acceptable error level and 0.05 as the learning rate for the training. With this learning rate (0.05) and the goal error level (1x10-5), the training of the net has been completed. To complete the training it has taken 1627 epochs which has been shown in Fig. 3.1.2. The learning performance of the net as evident from the Fig. 3.1.2 in recognizing the data patterns is found to be $99.99 \%$.

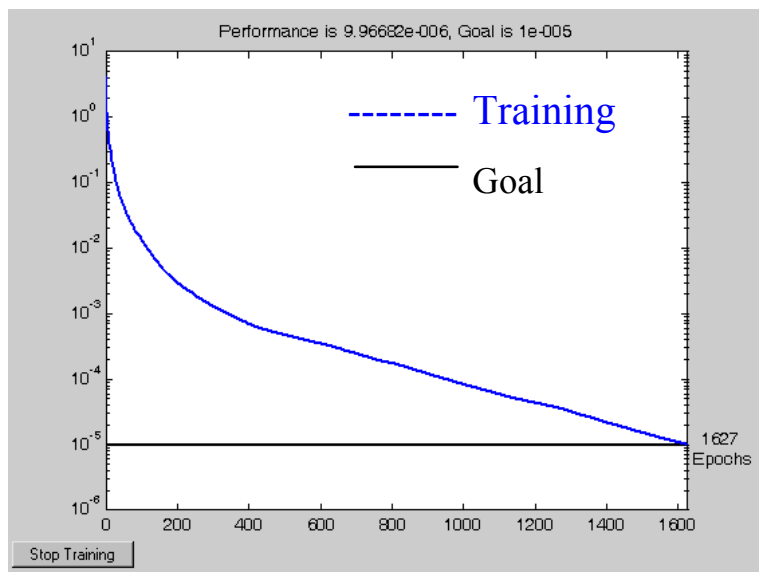

Fig. 3.1.2: Training of the Net ( SCGA)

Triangular Area Constraint (SCGA) : $\mathrm{x}_{\mathrm{j}}+\mathrm{y}_{\mathrm{j}} \leq \mathrm{c}$

After the training is completed, we have tested the prediction capabilities of the trained net by using 10 new data patterns. The predicted output values obtained from the net have been compared with those obtained analytically and it is found that they compare well within the acceptable limit. The predicted output from ANN model and the output obtained through Anal method are given in Table 3.1.2. Thus, it is evident from the above table that the network performance is very much encouraging for unseen samples Fok Hing Chi Tivive[9]. The Error percentages for $x_{1}, x_{2}, y_{1}$ and $y_{2}$ have been calculated using the formula given below and are given in Table 3.1.1.

Error percentage $=(($ Anal Value - ANN Value $) /$ Anal value)*100. 


\begin{tabular}{|c|c|c|c|c|}
\hline S.No & $\begin{array}{c}\mathbf{x}_{\mathbf{1}} \\
\text { \% Error }\end{array}$ & $\begin{array}{c}\mathbf{x}_{\mathbf{2}} \\
\text { \% Error }\end{array}$ & $\begin{array}{c}\mathbf{y}_{\mathbf{1}} \\
\text { \% Error }\end{array}$ & $\begin{array}{c}\mathbf{y}_{\mathbf{2}} \\
\text { \% Error }\end{array}$ \\
\hline 1. & 5.27 & 12.82 & 6.25 & 5.63 \\
\hline 2. & 4.33 & 2.81 & 4.20 & 1.49 \\
\hline 3. & 0.03 & 0.02 & 0.10 & 0.02 \\
\hline 4. & 1.84 & 2.29 & 1.91 & 1.21 \\
\hline 5. & 2.86 & 2.56 & 1.41 & 3.46 \\
\hline 6. & 4.73 & 6.47 & 5.23 & 3.64 \\
\hline 7. & 0.69 & 2.36 & 0.37 & 3.54 \\
\hline 8. & 12.46 & 1.86 & 3.31 & 3.40 \\
\hline 9. & 12.91 & 3.65 & 5.32 & 8.13 \\
\hline 10. & 0.21 & 2.34 & 0.65 & 1.25 \\
\hline
\end{tabular}

Table 3.1.1 : Error Percentage

The final output locations $(\mathrm{x} 1, \mathrm{y} 1)$ and $(\mathrm{x} 2, \mathrm{y} 2)$ are given below in Table 3.1.2

\begin{tabular}{|c|c|c|c|c|}
\hline \multirow{2}{*}{} & \multicolumn{2}{|c|}{$\left(\mathbf{x}_{\mathbf{1}}, \mathbf{y}_{\mathbf{1}}\right)$} & \multicolumn{2}{c|}{$\left(\mathbf{x}_{\mathbf{2}}, \mathbf{y}_{\mathbf{2}}\right)$} \\
\cline { 2 - 5 } & Anal & $\mathbf{A N N}$ & Anal & $\mathbf{A N N}$ \\
\hline \multirow{2}{*}{1.} & $(2.17$, & $(2.13$, & $(1.22$, & $(1.28$, \\
& $1.83)$ & $1.87)$ & $2.78)$ & $2.72)$ \\
\hline \multirow{2}{*}{2.} & $(2.17$, & $(2.15$, & $(0.80$, & $(0.77$, \\
& $1.83)$ & $1.85)$ & $3.20)$ & $3.23)$ \\
\hline \multirow{2}{*}{3.} & $(1.63$, & $(1.65$, & $(2.43$, & $(2.54$, \\
& $3.37)$ & $3.35)$ & $2.57)$ & $2.46)$ \\
\hline \multirow{2}{*}{4.} & $(3.91$, & $(3.89$, & $(2.71$, & $(2.71$, \\
& $1.09)$ & $1.11)$ & $2.29)$ & $2.29)$ \\
\hline \multirow{2}{*}{5.} & $(3.05$, & $(3.08$, & $(2.07$, & $(2.08$, \\
& $2.95)$ & $2.92)$ & $3.93)$ & $3.92)$ \\
\hline \multirow{2}{*}{6.} & $(1.98$, & $(1.82$, & $(3.45$, & $(3.38$, \\
& $4.02)$ & $4.18)$ & $2.55)$ & $2.62)$ \\
\hline \multirow{2}{*}{7.} & $(1.05$, & $(1.03$, & $(0.72$, & $(0.73$, \\
& $0.95)$ & $0.97)$ & $1.28)$ & $1.27)$ \\
\hline \multirow{2}{*}{8.} & $(1.41$, & $(1.11$, & $(4.61$, & $(4.76$, \\
& $4.59)$ & $4.55)$ & $1.39)$ & $1.24)$ \\
\hline \multirow{2}{*}{9.} & $(1.75$, & $(1.45$, & $(3.00$, & $(3.05$, \\
& $3.25)$ & $3.32)$ & $2.00)$ & $1.95)$ \\
\hline \multirow{2}{*}{10.} & $(1.26$, & $(1.68$, & $(3.88$, & $(3.80$, \\
& $4.74)$ & $4.89)$ & $2.12)$ & $2.20)$ \\
\hline
\end{tabular}

The graphical representations of the values of $\mathrm{x} 1$ and $\mathrm{x} 2$ obtained analytically and through ANN approach have been shown below in Fig. 3.1.3 and Fig. 3.1.4 respectively.

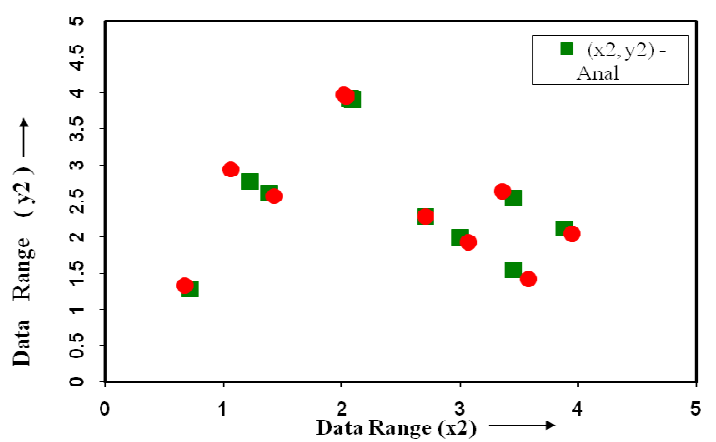

Fig. 3.1.3: $\left(\mathrm{x}_{1}, \mathrm{y}_{1}\right)-$ Anal vs ANN

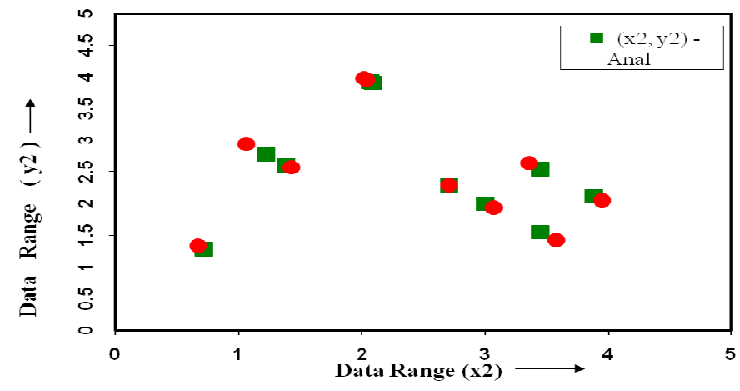

Fig 3.1.4: $\left(\mathrm{x}_{2,} \mathrm{y}_{2}\right)-$ Anal vs ANN

\section{CONCLUSION}

The present work involves developing the solutions for any given multifacility location problem under triangular area constraints using ANN technique and, comparing the results with the solutions obtained analytically first time in the literature. The capability of ANN technique to produce acceptable solutions for MLP's with triangular constraints within the acceptable limits of errors has been established.

\section{REFERENCES}

[1] Cabot A.V., Francis R.L. and Stary M.A., AIIE Transactions, Vol. 2, No. 2, June 1970 p.132.

[2] Pritsker A.A.B., and Ghare P.M., AIIE Transactions, Vol. 2, No. 4, December 1970, p.290.

[3] Kuhn H.W., and Kuenne R.E., Journal of Regional Science, 4, p. 21-33, (1962).

[4] Eyster J.W., and White J.A., AIIE Transactions, Vol. 5, No. 3, p. 275. (September 1973).

[5] McHose A.H., The journal of Industrial Engineering, Vol. 12, No. 5, Sept. - Oct., 1961, p. 334.

[6] Moller, M.F., "Learning by Conjugate Gradients", the 6th International Meeting of Young Computer Scientists, Czechoslovakia, in press, 1990

[7] Rumelhart, D.E., G.E. Hinton, R.J. Williams "Learning Internal Representations by Error Propagation”, in: Parallel Distributed Processing: Exploration in the Microstructure of Cognition, Eds. D.E. Rumelhart, J.L. McClelland, MIT Press, Cambridge, MA., pp. 318- 362, 1986.

[8] Da-Zheng Feng, Xian-Da Zhang, and Zheng Bao, "A Neural Network Learning for Adaptively Extracting Cross-Correlation Features between two High- Dimensional Data Streams", Neural Networks, IEEE, Vol. 15, No. 6, pp. 1541-1554, 2004.

[9] Fok Hing Chi Tivive, and A. Bouzerdoum, "Efficient Training Algorithms for a Class of Shunting Inhibitory Convolutional

Neural Networks", Neural Networks, IEEE, Vol. 16, No. 3, pp. 541-556, 2005. 